University of New Hampshire

University of New Hampshire Scholars' Repository

Space Science Center

Institute for the Study of Earth, Oceans, and

Space (EOS)

$11-21-2000$

\title{
Three-dimensional imaging and detection efficiency performance of orthogonal coplanar CZT strip detectors
}

\author{
Mark L. McConnell \\ University of New Hampshire - Main Campus, mark.mcconnell@unh.edu \\ John R. Macri \\ University of New Hampshire - Main Campus, John.Macri@unh.edu \\ James M. Ryan \\ University of New Hampshire, James.Ryan@unh.edu \\ K Larson \\ University of New Hampshire - Main Campus \\ L A. Hamel \\ University of Montreal
}

See next page for additional authors

Follow this and additional works at: https://scholars.unh.edu/ssc

Part of the Astrophysics and Astronomy Commons

\begin{abstract}
Recommended Citation
Mark L. McConnell ; John R. Macri ; James M. Ryan ; Kipp Larson ; Louis-Andre Hamel ; G. Bernard ; C. Pomerleau ; Olivier Tousignant ; Jean-Charles Leroux and Valentin T. Jordanov "Three-dimensional imaging and detection efficiency performance of orthogonal coplanar CZT strip detectors", Proc. SPIE 4141, Hard X-Ray, Gamma-Ray, and Neutron Detector Physics II, 157 (November 21, 2000); doi:10.1117/ 12.407576; http://dx.doi.org/10.1117/12.407576
\end{abstract}

This Conference Proceeding is brought to you for free and open access by the Institute for the Study of Earth, Oceans, and Space (EOS) at University of New Hampshire Scholars' Repository. It has been accepted for inclusion in Space Science Center by an authorized administrator of University of New Hampshire Scholars' Repository. For more information, please contact Scholarly.Communication@unh.edu. 


\section{Authors}

Mark L. McConnell, John R. Macri, James M. Ryan, K Larson, L A. Hamel, G Bernard, C Pomerleau, 0 Tousignant, Jean-Charles Leroux, and $\mathrm{V} \mathrm{T}$. Jordanov 


\title{
Three-dimensional imaging and detection efficiency performance of orthogonal coplanar CZT strip detectors
}

\author{
M. L. McConnell ${ }^{*}$, J. R. Macria ${ }^{\mathrm{a}}$ J. M. Ryan ${ }^{\mathrm{a}}$, K. Larson ${ }^{\mathrm{a}}$, L.-A. Hamel ${ }^{\mathrm{b}}$, \\ G. Bernard ${ }^{\mathrm{b}}$, C. Pomerleau, O. Tousignant, J.-C. Leroux ${ }^{\mathrm{b}}$, and V. Jordanov ${ }^{\mathrm{c}}$ \\ ${ }^{\mathrm{a} S}$ Space Science Center, University of New Hampshire, Durham, NH 03824 USA \\ ${ }^{\mathrm{b}}$ Physics Department, University of Montreal, Montreal, Quebec, Canada \\ ${ }^{c}$ Yantra, Durham, NH 03824 USA
}

\begin{abstract}
We report on recent three-dimensional imaging performance and detection efficiency measurements obtained with $5 \mathrm{~mm}$ thick prototype CdZnTe detectors fabricated with orthogonal coplanar anode strips. In previous work, we have shown that detectors fabricated using this design achieve both very good energy resolution and sub-millimeter spatial resolution with fewer electronic channels than are required for pixel detectors. As electron-only devices, like pixel detectors, coplanar anode strip detectors can be fabricated in the thickness required to be effective imagers for photons with energies in excess of 500 $\mathrm{keV}$. Unlike conventional double-sided strip detectors, the coplanar anode strip detectors require segmented contacts and signal processing electronics on only one surface. The signals can be processed to measure the total energy deposit and the photon interaction location in three dimensions. The measurements reported here provide a quantitative assessment of the detection capabilities of orthogonal coplanar anode strip detectors.
\end{abstract}

Keywords: CdZnTe, CZT, solid-state radiation detectors, imaging X-ray detectors, X-ray spectroscopy, gamma-ray spectroscopy, X-ray astronomy, gamma-ray astronomy, detector packaging

\subsection{INTRODUCTION}

Cadmium Zinc Telluride (CZT) detectors are well suited for fabrication of large area, high performance X-ray and $\gamma$-ray imaging spectrometers. They have the desirable properties of high stopping power, low thermal noise, room-temperature operation, excellent energy resolution and unsurpassed spatial resolution. With current technologies, high performance CZT imaging spectrometers can be adapted for use in a variety of imaging techniques to image photons at energies up to a few hundred $\mathrm{keV}$. In the future, as the quality of CZT material improves to the point where the use of thicker detector substrates become feasible, the useful energy range of CZT detectors will likely be extended into the MeV energy range.

Many high-energy astrophysical observations require better energy resolution than can be obtained with scintillators, but do not necessarily require the energy resolution of germanium with all its attendant complexities, in particular the cryogenic requirements. These cryogenic needs for germanium spectrometers take the form of LN2 or refrigerators that add cost, mass and complexity to the instrument. Excellent energy and spatial resolution can, however, be achieved with CZT detectors operating at room temperatures.

The spectroscopic value of CZT is exemplified by its use in NASA's upcoming SWIFT mission. ${ }^{1}$ Related CdTe detectors are also being deployed on the INTEGRAL mission of ESA. ${ }^{2}$ Neither of these applications take advantage of the properties of CZT that allow for the determination of a photon interaction site within a detector. Instead, small detector elements serve to isolate the location of the photon capture to within several millimeters. Outfitting large areas with numerous small detector elements so that the improved (sub-mm) spatial resolution could be achieved would be prohibitively complex and expensive. The only practical solution would be to employ large-area position-sensitive detectors, drastically reducing the number of detectors and associated electronic channels.

\footnotetext{
*Correspondence:E-mail: Mark.McConnell@unh.edu; telephone: 603-862-2047; fax: 603-862-4685
} 
An image plane fabricated using closely-packed arrays of CZT detector modules is a candidate for the central detector of several proposed hard X-ray $(30-600 \mathrm{keV})$ coded aperture telescopes, including HEXIS (High-Energy X-ray Imaging Spectrometer), ${ }^{3}$ AXGAM (All-sky X-ray an Gamma-ray Astronomy Monitor), ${ }^{4}$ EXIST (Energetic X-Ray Imaging Survey Telescope), ${ }^{5}$ and MARGIE (the Minute-of-Arc Resolution Gamma-ray Imaging Experiment). ${ }^{6,7}$ In order to achieve angular resolutions on the order of one arc-minute (the design goal of the MARGIE program, for example), the detectors for the image plane will require sub-millimeter position resolution in three dimensions. Energy resolutions of better than $10 \%$ FWHM at $60 \mathrm{keV}$ are desired for these applications. CZT strip detectors are also under investigation for use as calorimeter detectors in an innovative new Compton telescope design called TIGRE (and the Tracking and Imaging Gamma-Ray Experiment). ${ }^{8}$ TIGRE is intended to operate in the range of $100 \mathrm{keV}$ to $100 \mathrm{MeV}$. CZT represents a promising alternative to germanium strip detectors or scintillators for reasons of performance and complexity. The position resolution goal for the TIGRE calorimeter is $\pm 1 \mathrm{~mm}$ in each of 3 dimensions. The energy resolution goal for the calorimeter is $0.7 \%$ FWHM at 662 $\mathrm{keV}$. Both MARGIE and TIGRE will require image planes with areas exceeding $1000 \mathrm{~cm}^{2}$. Both are currently being studied as concepts for Ultra-Long Duration Balloon (ULDB) payloads and subsequent space flight experiments.

Large area CZT image planes would also be valuable tools in a variety of industrial, scientific and defense applications. They would, for example, significantly enhance performance in medical imaging applications such as mammography and SPECT. CZT detectors have been undergoing an intensive development effort by researchers in all these fields because of their desirable properties.

In earlier work, we reported on studies of double-sided CZT strip detectors with orthogonal anode and cathode strips. ${ }^{9-13}$ In those publications we recognized that the poor transport of the hole charge carriers restricts the effective thickness of detectors that have their signal contacts on both the anode and cathode surfaces. In double-sided strip detectors events interacting far from the cathode may not generate large enough signals to trigger the cathode strip electronics to identify the second coordinate of the interaction location. Our recent work has concentrated on developing a device based on an orthogonal coplanar anode strip design. ${ }^{14-18}$ This approach deals effectively with the problem of poor hole transport in CZT that severely limits the usefulness of traditional double-sided strip detectors. It achieves excellent energy resolution and offers the potential for using much thicker detector substrates. This, in turn, provides greater detection efficiency at higher energies $(>500 \mathrm{keV})$, while maintaining the capability of sub-mm spatial resolution in three spatial dimensions. Furthermore, we have demonstrated a prototype packaging concept based on polymer flip-chip bonding that eliminates all wire bonds and permits the fabrication of large area imaging arrays with large packing fraction. We fabricated and tested the first prototypes of this design. Most recently, ${ }^{17,18}$ we reported on results from energy resolution measurements and from studies of event location resolution using a collimated alpha source. Here, we present our latest test results (including measurements of the photon interaction depth and mean free path), an overview of detector signal characteristics and how we plan to use them for depth measurements, and our latest concept for fabricating a closely-packed array of detector modules.

\subsection{THE ORTHOGONAL COPLANAR ANODE STRIP DETECTOR}

Good efficiency, energy resolution and position resolution have been demonstrated in pixellated CZT detectors up to $10 \mathrm{~mm}$ thick. ${ }^{19}$ These detectors are electron-only devices that avoid the deleterious consequences of poor hole transport. Unfortunately, the anode pixel contact geometry requires an electronic signal channel for each pixel, $\mathrm{N}^{2}$ channels in an $\mathrm{N}$ row $\times \mathrm{N}$ column array. Strip detectors, on the other hand, provide $\mathrm{N}^{2}$ pixels with only $2 \mathrm{~N}$ electronic channels, one for each row and one for each column. This is important in space flight applications where channel count greatly affects the power requirements, packaging, thermal design and complexity of the instrument electronics. Double-sided CZT strip detectors, if carefully designed, can address much of the limitation associated with poor hole transport. ${ }^{20}$ There will, however, always be an upper limit on detector thickness $(\sim 3 \mathrm{~mm})$ and thus the effective energy range $(<300 \mathrm{keV})$. Double-sided strip detectors also have the requirement that electronic signals must be processedfrom the strip contacts on both surfaces. The wiring associated with these contacts adds to dead area in closely packed arrays of imaging modules.

To address these problems and to explore new fabrication technologies we have been investigating and developing a novel CZT detector concept: an electron-only device featuring orthogonal coplanar anode strips. Each row takes the form of $\mathrm{N}$ discrete interconnected anode pixels while each column is a single anode strip. Figure 1 illustrates the anode surface contact 


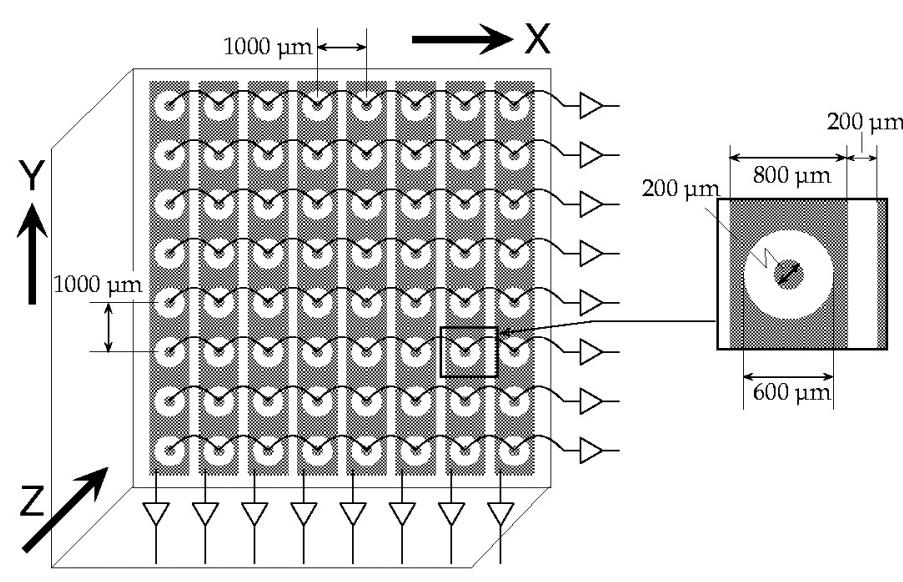

Figure 1. A schematic diagram showing the layout and read out of the orthogonal coplanar anode design. Strip columns $(\mathrm{X})$ are read out on the bottom. Pixel rows $(Y)$ are read out on the right. The gold contact pattern dimensions shown here in grey correspond to those of our prototype assemblies. Prototype CZT thickness is $5 \mathrm{~mm}$. pattern and the readout of an $8 \times 8$ pixel orthogonal coplanar anode strip detector. The opposite side, not shown, has a single uniform cathode electrode. The anode pixel contacts, interconnected in rows, are biased to collect the electron charge carriers. The orthogonal anode strips, surrounding the anode pixel contacts, are biased between the cathode and anode pixel potentials. The strips register signals from the motion of electrons as they migrate to the pixels. Since electrons are much more mobile than holes in CZT, signals from photon interactions at all depths in the detector are detected. Given the published results with pixel detectors ${ }^{19}$, we expect our coplanar anode strip approach to be effective in CZT detectors at least $10 \mathrm{~mm}$ thick. This will permit thicker, more efficient, CZT imaging planes than are practical with doublesided strip detectors and will extend the effective energy range to $>1 \mathrm{MeV}$. In addition more compact packaging is possible since all imaging contacts and signal processing electronics connections are on only one side of the detector. Thus, one can expose the cathode surface to the incident photon flux permitting closer packing of imaging CZT modules in an array.

\subsection{ASSEMBLY OF PROTOTYPE MODULES}

Our prototype module assemblies involve the application of two key technologies: Low Temperature Cofired Ceramics (LTCC) and Polymer Flip-Chip (PFC) bonding. ${ }^{21}$ Figure 2 is a photograph of prototype components: a patterned CZT substrate (left) and its mating LTCC carrier. Assembly involves electrical and mechanical PFC bonding between the two surfaces shown in the photograph. The $12 \mathrm{~mm}$ square by $5 \mathrm{~mm}$ thick substrate for our prototype detector is discriminatorgrade single-crystal CZT material from eV Products. Anode pixel contact pads are $200 \mu \mathrm{m}$ in diameter and the pitch is $1 \mathrm{~mm}$ in each dimension. Note the pattern of 8 rows of gold pixel contacts and 8 gold strips that form 64 pixels on the anode

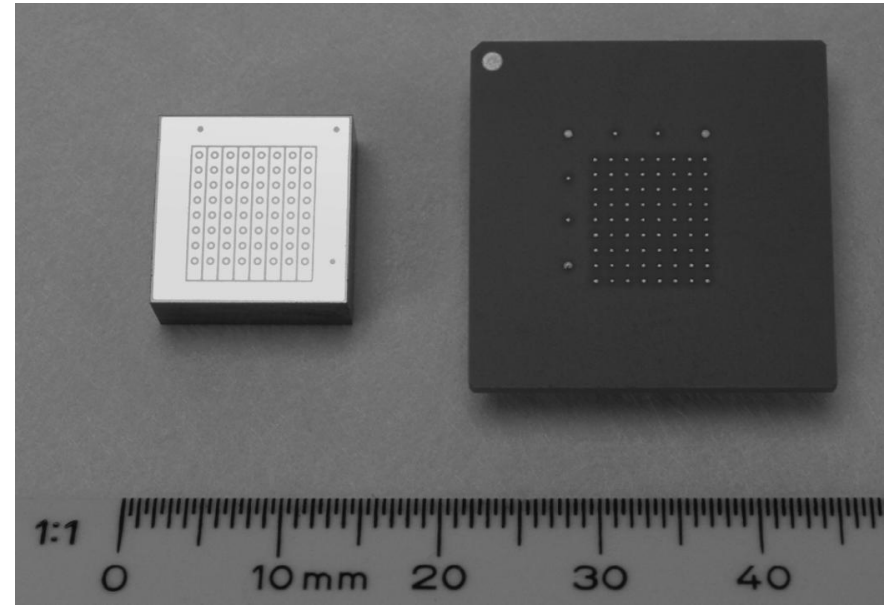

Figure 2. Detector prototype components: a patterned CZT substrate (left) and its mating ceramic (LTCC) carrier. The light-colored areas on the surfaces are gold contacts.

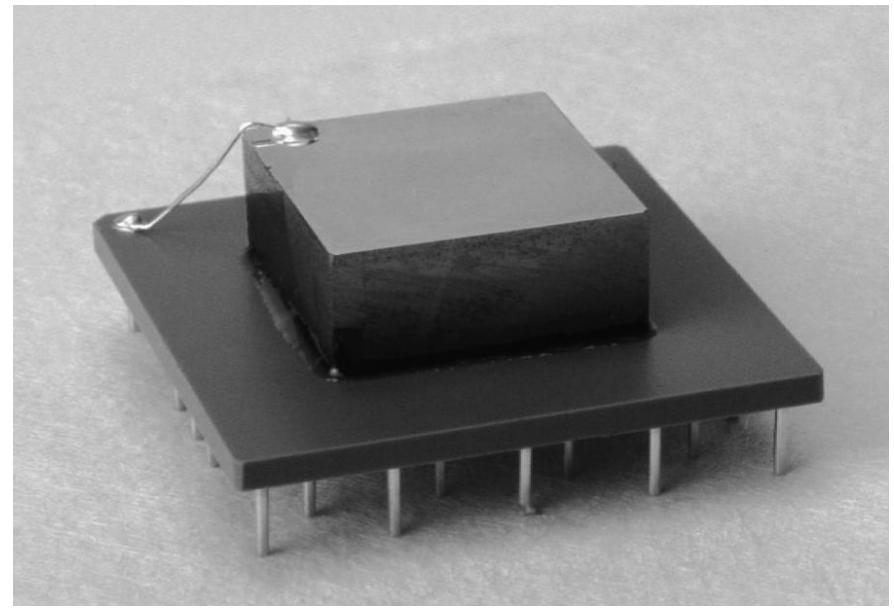

Figure 3. The first prototype detector assembly. A $5 \mathrm{~mm}$ thick patterned CZT substrate is PFC-bonded to its mating LTCC substrate. 
surface and the fiducial marks in the guard ring area. The $12 \mathrm{~mm}$ square dimension was selected for the first prototypes because the manufacturer was making detectors in this dimension for another customer and this sized substrate was therefore available at reduced cost. Note that the guard ring can be much smaller or even eliminated in future detectors.

On the right in Figure 2 is the mating LTCC substrate for connecting the module to the support electronics. There are 8 rows of 8 gold contact pads corresponding to the pixels on the CZT anode. There is a ninth row of 8 pads for making contact with the anode strips. A large contact pad in one corner connects the cathode bias. Interconnection of the pixels in rows and a breakout of the detector contact elements to pins in a standard pin grid array (PGA) pattern is on the underside of the LTCC substrate (not shown).

LTCC technology was chosen in part because it meets the fabrication requirements of the prototype assemblies: $170 \mu \mathrm{m}$ diameter filled vias with flat contact pads, low leakage under HV bias, and a thermal expansion coefficient similar to that of CZT. LTCC technology permits fabrication of multi-chip modules (MCM) having up to 100 layers with very reliable interconnections on pitches as fine as $300 \mu \mathrm{m}$. The technology also permits inclusion of mechanical fasteners for handling and assembly.

A new low temperature bonding process, Polymer Flip Chip (PFC) bonding ${ }^{22,23}$, is well suited to the special low temperature handling requirements for CZT. It was used to form the electrical and mechanical connection of the patterned CZT and LTCC substrates of our prototype detector assemblies. The PFC bonding technique utilizes silver-filled conductive epoxy to electrically connect the contacts of electronic components. Conducting polymer bumps $(\sim 120 \mu \mathrm{m}$ diameter, $\sim 20 \mu \mathrm{m}$ high $)$ are stencil printed and can be cured at temperatures as low as room temperature. All polymer bumps have uniform size and are well aligned with the $200 \mu \mathrm{m}$ diameter gold pixel contacts. Similar conducting bumps are stenciled on the mating LTCC substrates. The bumped surfaces are aligned, mated while one set of conducting bumps is still wet, then cured. The electrical interconnections established by the mated conducting bumps are very short, thus minimizing electronic noise. Contact resistance is insignificant, typically $10-20 \mathrm{~m} \Omega$.

A non-conducting epoxy underfill is then used to fill all voids between the mating surfaces and around the conducting bumps to provide mechanical strength. Temperatures $<80^{\circ} \mathrm{C}$ were used for all processing steps, well below the $100^{\circ} \mathrm{C}$ maximum recommended by CZT material manufacturers. Once the assembly is cured the rugged LTCC substrate can be used for all handling of the detector. The conducting bump and underfill materials are somewhat compliant and thus aid in reducing thermal and mechanical stress to the CZT. Furthermore, the underfill provides thermal isolation from the electronics. The result is a rugged assembly that involves no wire bonds to the CZT anode surface (Figure 3). Once set up, this bonding process is fully automated and produces highly reliable interconnections at low incremental cost. PFC bonding is used in other applications to reliably interconnect pads as small as $75 \mu \mathrm{m}$ with pitch as fine as $125 \mu \mathrm{m}$. Details of the application of this technology to our prototype CZT module have been previously published. ${ }^{21}$

We have demonstrated the feasibility of the electrical and mechanical PFC bonding technique in balloon and space applications by subjecting a prototype module to thermal cycle and vibration tests. A prototype detector assembly was cycled in the temperature range $-20^{\circ} \mathrm{C}$ to $+50^{\circ} \mathrm{C}$ at a rate of $20^{\circ} \mathrm{C} / \mathrm{hr}$. It was then exposed to random and structural vibration in the thrust and lateral axes according to qualification levels appropriate for an instrument launched on a Delta II vehicle. We measured all pixel and strip channels before and after these environmental exposures and observed no significant changes.

\subsection{PROTOTYPE PERFORMANCE}

\subsection{Spectroscopy}

The spectroscopic performance of a single pixel element is demonstrated in Figure 4, which shows energy deposit spectra collected with one of the first prototype detector assemblies. The detector was operated with the cathode at $-800 \mathrm{~V}$, the anode pixels at $0 \mathrm{~V}$ and the anode strips at $-30 \mathrm{~V}$. The measured leakage current under these conditions is $3.5 \mathrm{nA}$. The test pulse data shown for two of these spectra indicate that electronic noise presently limits the energy resolution and suggests that further improvements may still be possible. This claim is supported by a preliminary noise analysis involving both lab measurements and simulations. 

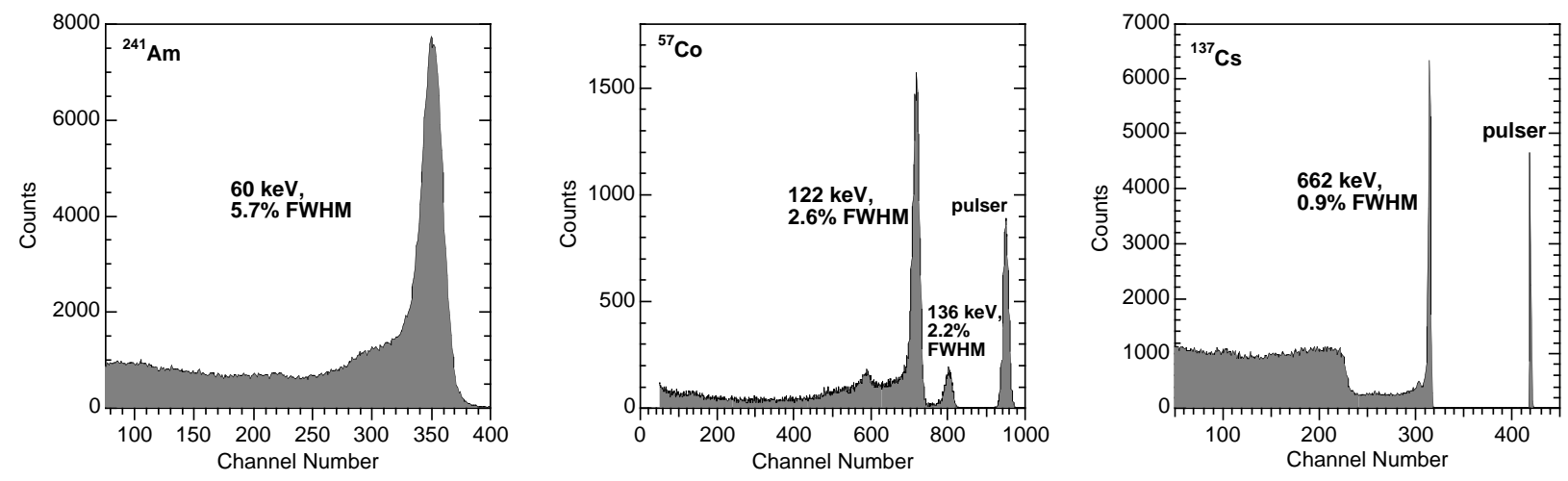

Figure 4. Single pixel response to calibration photons from ${ }^{241} \mathrm{Am}$ (left), ${ }^{57} \mathrm{Co}$ (center), and ${ }^{137} \mathrm{Cs}$ (right).
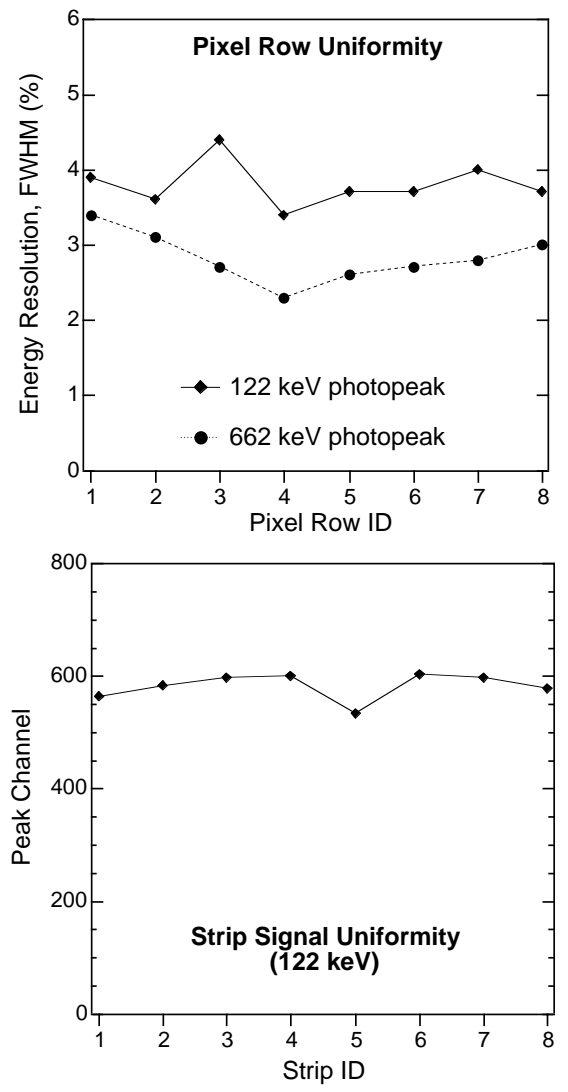

Figure 5. Measurements of the signal uniformity. TOP: Energy resolution at both $122 \mathrm{keV}$ and $662 \mathrm{keV}$ as a function of pixel row. BOTTOM: Gain uniformity (in terms of peak location) as measured at $122 \mathrm{keV}$ for each strip.
Measurements of the detection uniformity are presented in Figure 5. The measured energy resolution at $122 \mathrm{keV}$ and $662 \mathrm{keV}$ for each row of interconnected anode pixels is shown in the top panel of Figure 5. Similarly, the measured variation of signal pulse heights among the orthogonal strips is shown in the bottom panel of Figure 5. Note that since no pixel-strip coincidence was required, the energy resolution is somewhat worse for an entire pixel row than it is for a single pixel (cf., Figure 4). The important point to be made here is that the detector fabrication and bonding techniques yield reliable connections to all 64 pixels of the detector.

\subsection{Measurements of interaction site location}

The $\mathrm{X}$ and $\mathrm{Y}$ spatial location capabilities of the prototype detectors have been previously demonstrated using scans in $100 \mu \mathrm{m}$ steps across a representative region of the detector with a collimated alpha source incident on the cathode surface. ${ }^{17,18}$ Some charge sharing occurs in both directions, although the level of charge sharing in the X-direction is somewhat higher. This increase in charge sharing results from the larger area of the strip, which makes it sensitive to electron motion at greater distances. Some level of charge sharing is required in order to infer locations from relative signal measurements. In this case, better location information can be inferred in the X-direction (perpendicular to the strips). The alpha scan measurements have yielded $1 \sigma$ spatial resolutions of $\sim 100 \mu \mathrm{m}$ in $\mathrm{X}$ and $\sim 300 \mu \mathrm{m}$ in $\mathrm{Y}$.

The interaction depth $(Z)$ is presently inferred from the ratio of the amplitudes of the cathode and of the largest pixel signal and, in our protype modules, is equal to $(1-$ cathode/pixel $) \times L$, where $L=5 \mathrm{~mm}$ is the detector thickness. Figure 7 shows the results of a simple interaction depth measurement that employed a tungsten sheet as a shield for $122 \mathrm{keV}$ photons incident from the side of the detector. The shield was positioned in two locations differing in $\mathrm{Z}$ by $0.5 \mathrm{~mm}$. The distribution in Figure 7 represents the difference between the interaction depth profiles measured for the two shield positions. This effectively corresponds to the distribution of events entering the CZT from the side through a $500 \mu \mathrm{m}$ slit. The width of the measured distribution shown in Figure 7, considering the $500 \mu \mathrm{m}$ shift of the tungsten shield, corresponds to a $1 \sigma \mathrm{FWHM}$ position resolution of $650 \mu \mathrm{m}$ in the Z-coordinate. 


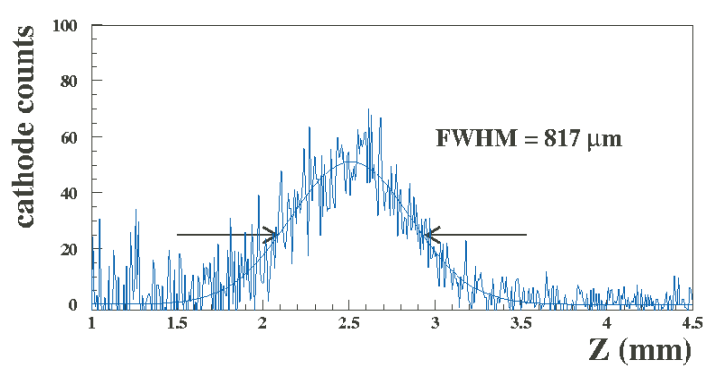

Figure 7. Z-position resolution for $122 \mathrm{keV}$ photons using the ratio of cathode and anode pixel signals.
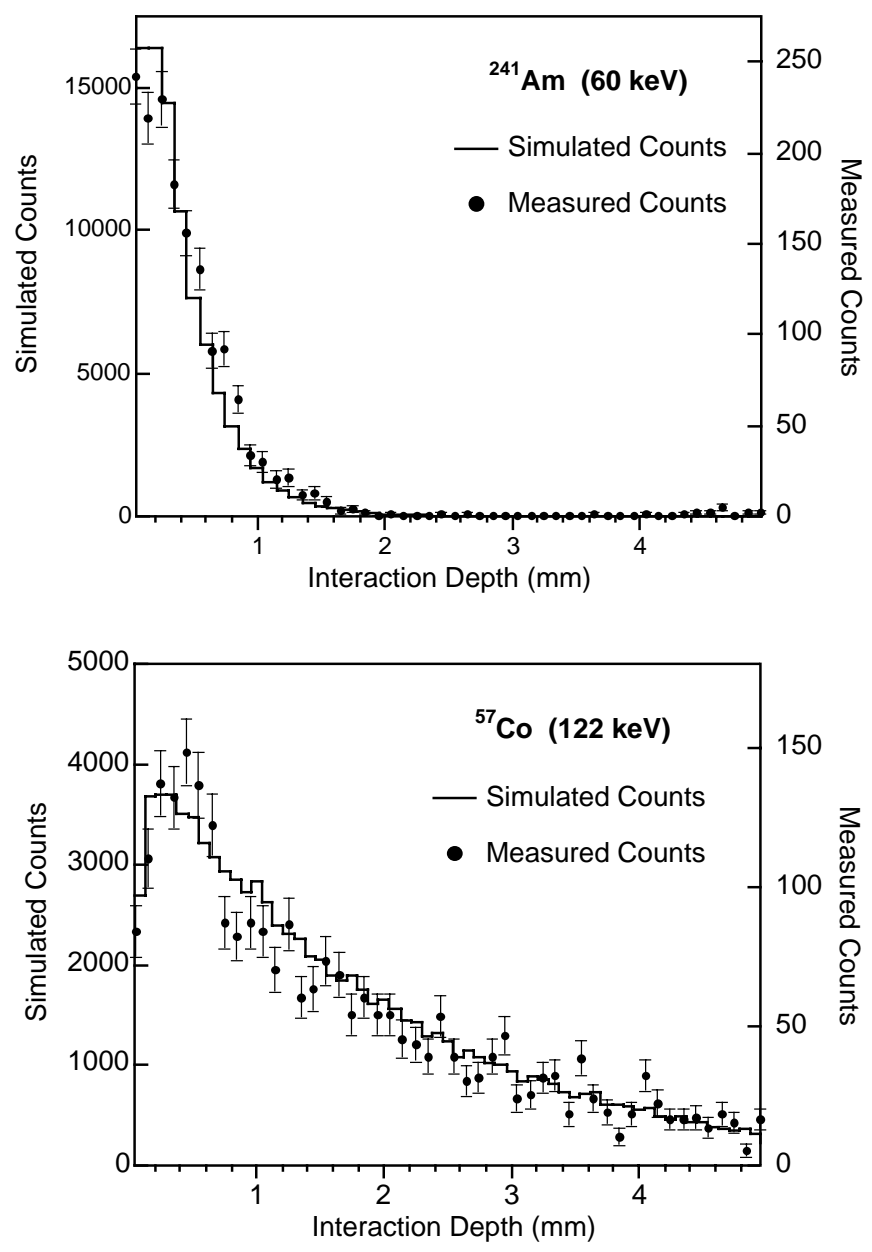

Figure 8. Profiles of interaction depths for incident photon energies of $60 \mathrm{keV}$ (top) and $122 \mathrm{keV}$ (bottom).
A profile of interaction depths can be used to determine the attenuation length of photons in CZT, from which a measure of the detection efficiency can be obtained. Figure 8 shows the profile of interaction depths for incident photons of 60 $\mathrm{keV}$ and $122 \mathrm{keV}$. The cathode to anode pulse height ratio was used for these measurements. Also shown are simulated profiles based on simulations with GEANT. (The GEANT simulations also include the escape of X-ray fluorescence photons for photoelectric interactions near the cathode surface, the effect of which is especially apparent in the 122 $\mathrm{keV}$ data.) Fits to the data give attenuation lengths of $0.42 \pm 0.02 \mathrm{~mm}$ at $60 \mathrm{keV}$ and $1.72 \pm 0.15 \mathrm{~mm}$ at $122 \mathrm{keV}$. These values compare favoarably with tabulated values of $0.27 \mathrm{~mm}$ and $1.65 \mathrm{~mm}$, respectively. This further reinforces our confidence in the ability to measure photon interaction depths.

While the cathode signal can be useful for the depth of interaction measurement, the need to feed the cathode signals from the front surface of the detector to front-end electronics located behind the detector will interfere with the fabrication of closely packed arrays of detector modules. For this reason, we have been studying approaches that will permit the measurement of the interaction depth using signal processing electronics on only the anode pixel row and strip contacts, both of which are on the same (back) surface of the detector and would be readily accessible to the front-end electronics. ${ }^{24}$ Figure 9 illustrates the basic features of both the anode strip and anode pixel signals (the charge sensitive preamplifier outputs). The pixel signals, rising in only the positive direction, are typical of small-pixel anodes in CZT detectors. The initial slope of the pixel signals is small but increases rapidly when electrons reach the anode region. These signals provide a measure of the energy deposit and identify the $\mathrm{Y}$-coordinate of the photon interaction location. The strip signals identify the X-coordinate of the photon interaction location, but are not used for an energy measurement. The strip signals are bipolar in nature. They have faster initial rises than the pixel signals due to the larger strip areas. They reach a maximum shortly before electron transit time and decrease as the electrons approach the pixel. Of particular interest are three features of the anode strip signal (risetime, time-over-threshold and residual) that can be used to measure the depth of interaction, independent of any signal from the cathode. Figure 10 shows simulated results summarizing the relationship between these strip signal parameters and the interaction depth $(Z)$ for events occuring below the center of a pixel. Note that the strip signal risetime decreases with increasing interaction depth $(Z)$ and that the negative residual charge left on the strip at the end of the event is more negative at larger $Z$. This latter effect is due to trapped holes. These features of the strip signal can be exploited to extract the third spatial coordinate $(Z)$, the 


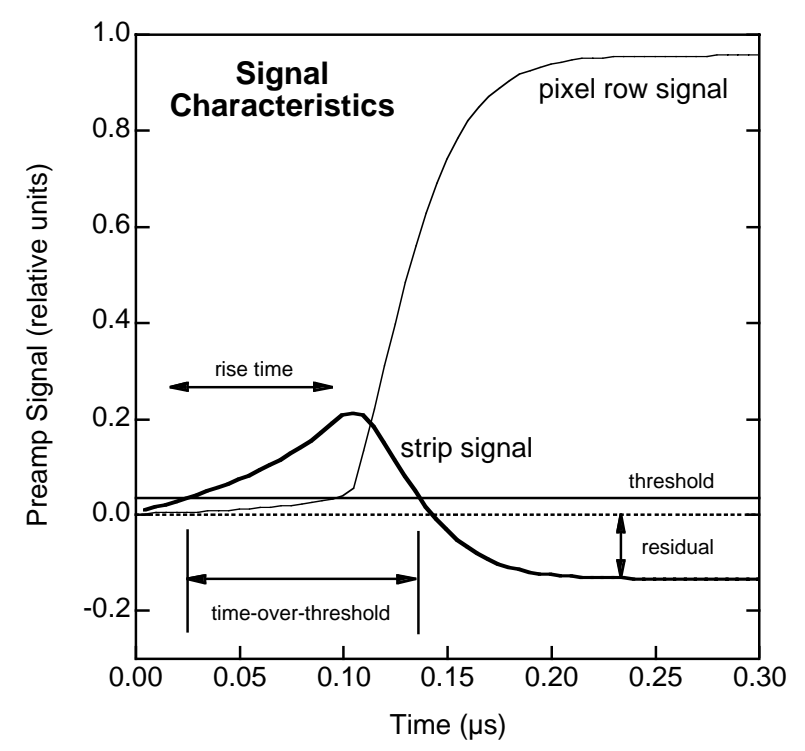

Figure 9. Characteristics of the strip and pixel row signals generated by a single interaction in the CZT.

depth of interaction.

Figure 11 shows data that demonstates the relationship to interaction depth for two of the strip signal parameters. The data were collected using $1.33 \mathrm{MeV}$ photons from a ${ }^{60} \mathrm{Co}$ source. For each event, the strip signal risetime is plotted versus the strip signal residual (cf., Figure 9), where the strip signal residual is measured relative to the residual of the pixel row signal. The solid line in Figure 7 represents the expected distribution as determined from simulations (cf., Figure 10). Some representative Z-coordinates are also indicated, based on the data in Figure 10.

We have been working to design and implement a circuit that can, using the strip signal as an input, directly measure the interaction depth. From our studies of the strip signal characteristics versus interaction depth, coupled with investigations of possible circuit designs, we have concluded that the time-over-threshold parameter (cf., Figure 9) represents the most promising approach for designing an effective, low power circuit. The latest results from a prototype time-over-threshold to amplitude conversion circuit (TOT-TAC) are being presented elsewhere. ${ }^{24}$

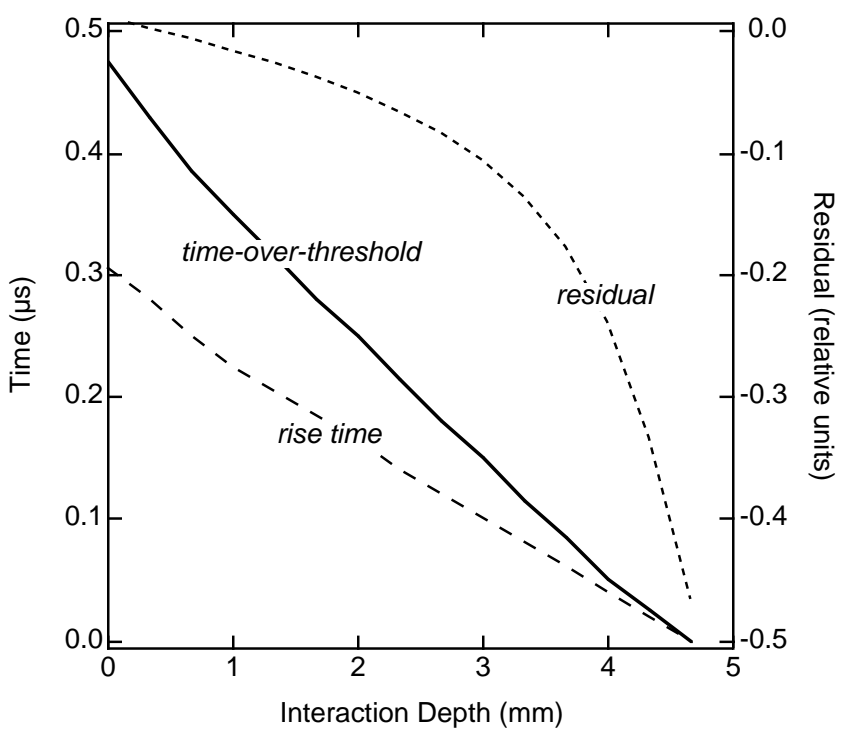

Figure 10. Simulated measurements of various strip signal parameters as a function of interaction depth.

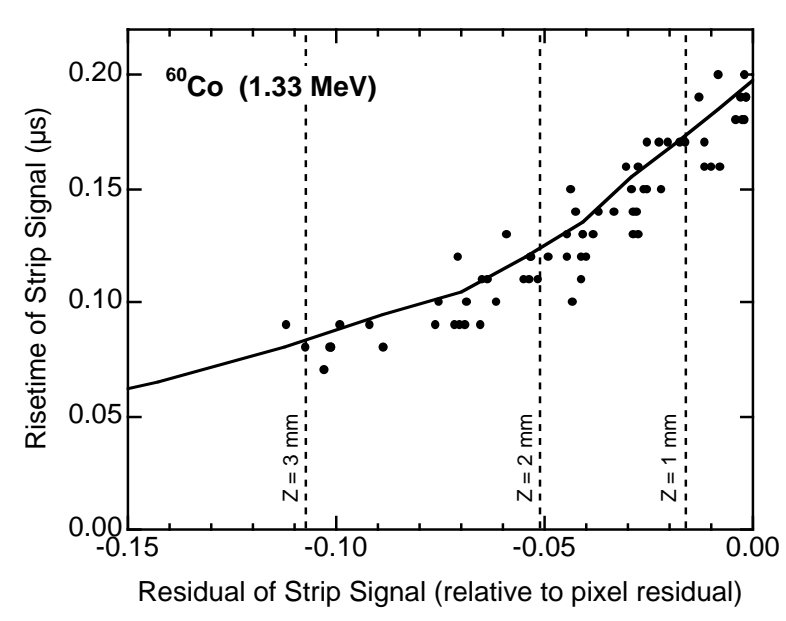

Figure 11. Measurements demonstrating the correlation between strip signal risetime and strip signal residual and their relationship to interaction depth (Z).

\subsection{Recent results with a VME-based data acquisition system}

The University of Montreal has recently designed and fabricated a data acquisition (DAQ) system to aid with the detailed study of this and other segmented electrode solid state detectors. The DAQ is VME- and FPGA-based and is controlled by a PC running LabView software. For our CZT prototype detector studies, the DAQ system supports 18 signal channels: 8 pixel rows, 8 orthogonal strips, the cathode and the guard ring. Each channel receives and simultaneously processes the outputs of the charge sensitive preamplifiers on each of the 18 detector electrodes. All signals are continuously sampled at $100 \mathrm{MHz}$ by 10 bit FADCs. The digitized traces are then processed by FPGAs using trapezoidal shaping. The shaping 
parameters are separately programmable on each channel; a base of 1 $\mu$ s and a flat top of $320 \mathrm{~ns}$ are presently used for the pixel channels. The DAQ system can be be self-triggered. It processes data on an event-byevent basis. In our application, a trigger is issued whenever a signal above threshold is detected in any pixel trapezoid at which time all channels are frozen. The digitized preamplifier and shaped traces are then available to the PC.

The DAQ system can be operated in a mode where it functions as an 18-channel digital oscilloscope, displaying and recording the preamplifier signal waveform for each detected event. This mode is particularly useful for studying signal shape and timing for events occurring at different locations within the detector volume. This provides important information to our electronics design effort.

In an imaging and spectroscopy mode, the DAQ system computes, for each event, one pulse height for each pixel row channel and one pulse height for each lobe (positive and negative) of each orthogonal strip channel. Only the measured pulse heights are then transferred to the PC. A more detailed description of this DAQ system will be published at a later date. The LabView program then computes, displays and records energy and position in three dimensions. The X-position (cf., Figure 1) is calculated from the pulse heights of the highest strip channel and its two neighbors and is equal to the $\mathrm{X}$-value of the highest channel plus or minus a correction that is proportional to the difference between the two neighbors. ${ }^{13}$ The Y-position is obtained from a

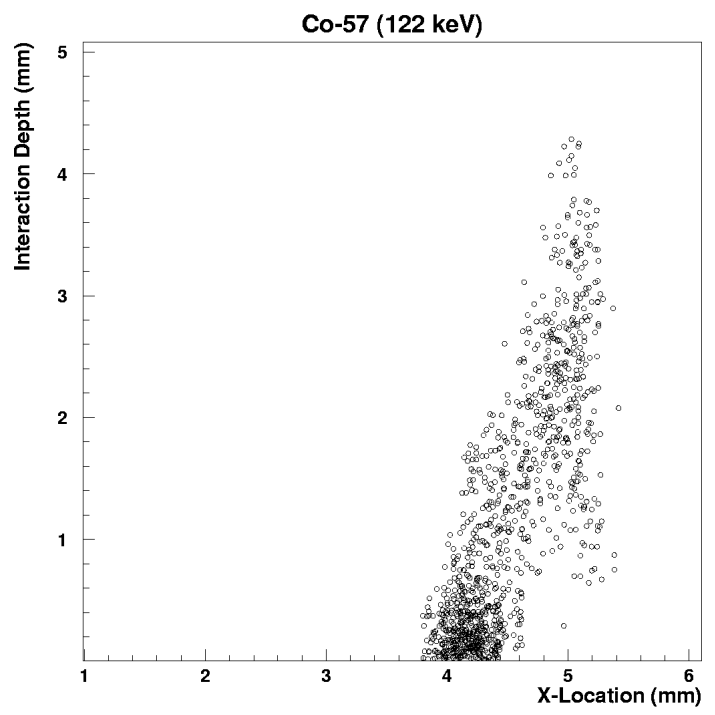

Figure 12. The distribution of $122 \mathrm{keV}$ interaction sites for a collimated beam obliquely incident on the front (cathode) surface of the detector (at $Z=0)$. In this view, the $100 \mu \mathrm{m}$-wide beam is incident from below and directed towards the upper-right.

weighted average of the amplitudes of the highest pixel signal and its two neighbors. The weighting is non-linear because of the limited signal sharing between adjacent pixel rows. ${ }^{13}$ The Z-coordinate is presently inferred from the ratio of the amplitudes of the cathode and of the largest pixel signal (see section 4.2$)$ and is equal to $(1-$ cathode/pixel)* $L$ with $L=5 \mathrm{~mm}$ is the detector thickness.

The first preliminary results from this DAQ system have recently become available. Figure 12 shows the distribution of ${ }^{57} \mathrm{Co}$ events $(122 \mathrm{keV})$ as measured using a collimated beam obliquely incident on the front (cathode) surface of the detector. The beam spot on the cathode surface is $\sim 100 \mu \mathrm{m}$ in diameter. As seen in Figure 12, the beam is incident from the bottom and diected towards the upper-right. The outline of the beam as it interacts within the CZT is clearly seen.

\subsection{FABRICATION CONCEPT FOR LARGE-AREA ARRAYS}

The baseline approach for packaging an imaging module is illustrated in Figure 13. A complete imaging module consists of a patterned CZT substrate that has been PFC bonded to a ceramic (LTCC) substrate or multi chip module (MCM). Here we envision a module with $16 \times 16$ logical pixels formed from 16 strips and 16 pixel rows, each on a $1 \mathrm{~mm}$ pitch. The CZT substrate in this case is roughly $16 \times 16 \mathrm{~mm}^{2}$.

The PFC bonding process establishes the mechanical and electrical interconnection between the CZT and LTCC substrates. The multi-layer LTCC substrate establishes the interconnection of the anode pixels in rows and the routing of the pixel row and strip signals (total of 32) plus the guard ring contact to flat gold contact pads on the underside of the module. Mechanical support for handling the module and aligning and mounting it to the image plane is integral to the LTCC substrate. The fact that 256 pixels are achieved with only 32 signal processing channels leaves ample space on the underside of the LTCC substrate for the passive components (resistors and capacitors) necessary for the ac-coupled strip signals and for the mounting hardware. Similarly, the limited number of channels permits ample space on the underside of the image plane board for each module's FEE ASICs within the module footprint. 


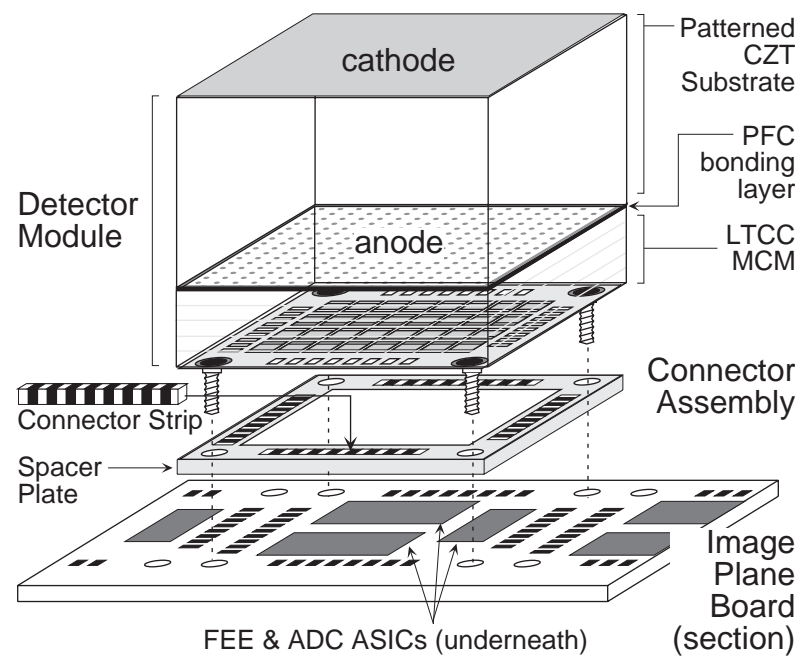

Figure 13. Concept for a detector module to be used in fabrication of large area arrays.
This modular concept has several important advantages:

- The rugged, compact design makes for easy handling and mounting of the CZT detectors.

- The CZT detector is thermally isolated from the heatgenerating electronics by the LTCC substrate and the PFC bonding layer.

- There is a clean separation between the passive and active components of the detection system without the need for long leads that not only take up space, but also add capacitance and noise.

- There can be a clean separation of the detector and front end electronics development efforts, without requiring costly and risky disassembly and re-assembly operations.

In Figure 14 we show the concept of an image plane board supporting a $20 \times 20 \mathrm{CZT}$ module array having $1024 \mathrm{~cm}^{2}$ active area. The image plane is a large area, mechanically reinforced circuit board that supports an array of closely packed CZT detector modules. Using $0.5 \mathrm{~mm}$ spacing between modules, a reasonable gap for facilitating assembly and disassembly, the estimated packing fraction may be as high as $94 \%$. The image plane board supports detector bias and the front end electronics ASICs and ADCs for pixel row and strip signal processing. These electronics are on the underside of the board. The PFC bonding and connector assembly interconnect technologies provide good thermal isolation between the image plane board and the CZT. The cathode bias will be provided using a thin flexible circuit located between rows of modules (not shown). A thin light-tight cover is also necessary.

An important advantage of the orthogonal coplanar anode strip design over designs incorporating pixel detectors is in the image plane power dissipation and its impact on the thermal design. In any solid state detector system, optimum performance requires close proximity of the FEE to the detector and that performance is degraded at higher operating temperatures. Assuming $2 \mathrm{~mW} /$ channel for the pixel and strip FEE ASICs, the total FEE power dissipation for the array shown in Figure 14 would be $26 \mathrm{~W}$. A pixel detector array of the same size would require $205 \mathrm{~W}$. In our design, the heat dissipated on the image plane-board is conducted to the experiment structure via its mounting frame. The electrical interfaces between the image plane and the image-plane controller are digital, thereby permitting flexibility in the location of the controller electronics.

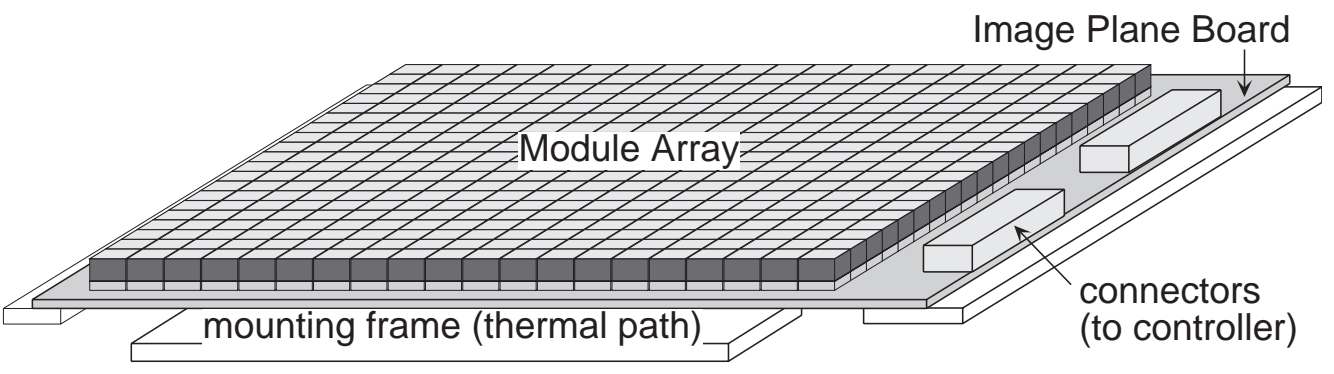

Figure 14. Image plane concept. A $20 \times 20$ array of imaging CZT modules. 


\subsection{FUTURE WORK}

Our efforts to date have served to demonstrate the viability of the orthogonal coplanar anode design. Measurements that are currently in progress will serve to more completely map out the spatial and energy response of a $5 \mathrm{~mm}$ thick prototype module. The development of a scheme to process only the anode signals (both pixel row and strip signals) will lead directly to the development of a suitable ASIC design, which will, in turn, lead to the fabrication of an engineering model based on the packaging concept presented in Figure 13.

A comparison between laboratory data and simulations has demonstrated the validity of the current simulation model. Although some improvements in the simulation model are being developed, we anticipate using the model to refine the anode pattern design. In particular, we will investigate variations in anode pitch, strip width, gap size between pixel and strip, and the extent to which a guard ring is needed. These studies will be incorporated into the fabrication of a thicker $(10 \mathrm{~mm})$ prototype detector. The value of CZT detectors for use in astrophysical applications would be greatly enhanced by the use of thicker detector substrates. The orthogonal co-planar anode design, being based on the collection of electrons only, offers the opportunity to fabricate much thicker strip detectors. The limiting thickness will ultimately depend on several factors, including the developments in fabricating large volume CZT crystals and the magnitude of electron trapping. In conjunction with these hardware efforts, we plan to perform detailed Monte Carlo studies to more fully characterize the physics of high energy $(>1 \mathrm{MeV})$ interactions in CZT. These studies will aid in extracting the maximum amount of information from events that undergo multiple interactions within the CZT material. Of particular importance for imaging applications will be the ability to identify the initial interaction site. This will be an important step in the evolution of high energy CZT detectors.

\section{REFERENCES}

1. SWIFT web site: http://swift.gsfc.nasa.gov.

2. F. Lebrun, C. Blondel, I. Fondeur, A. Goldwurm, P. Laurent, and J.-P. Leray, "ISGRI: A CdTe Array Imager for INTEGRAL," Proc. SPIE 2806, pp. 258-268, 1996.

3. J. L. Matteson, D. E. Gruber, W. A. Heindl, M. R. Pelling, L. E. Peterson, R. E. Rothschild, R. E. Skelton, W. R. Binns, P. L. Hink, and K. R. Slavis, "High-energy X-ray imaging spectrometer (HEXIS)," Proc. SPIE 3445, pp. 445-457, 1998.

4. V. V. Sushkov, W. J. Hamilton, K. Hurley, D. G. Maeding, H. Ogelman, R. J. Paulos, R. C. Puetter, T. O. Tümer, and J. Zweerink, "Preliminary Test Results of a New High Energy Resolution Silicon \& CdZnTe Pixel Detector for Application to X-Ray Astronomy," Proc. SPIE 3765, pp. 379-387, 1999.

5. J. Grindlay, L. Bildsten, D. Chakrabarty, M. Elvis, A. Fabian, F. Fiore, N. Gehrels, C. Hailey, F. Harrison, D. Hartmann, T. Prince, B. Ramsey, R. Rothschild, G. Skinner, and S. Woosley, "EXIST: A High Sensitivity Hard X-ray Imaging Sky Survey Mission for the ISS," Fifth Compton Symposium, eds. M.L. McConnell \& J.M. Ryan, AIP Conf. Proc. 510, pp. 784-7886. , AIP, New York, 2000.

6. M. L. McConnell, V. Boykin, R. M. Kippen, K. Larson, J. R. Macri, M. Mayer, J. M. Ryan, P. P. Altice, M. L. Cherry, S. B. Ellison, B. Price, G. T. Guzik, R. Lockwood, M. B. Barakat, ,K. Johnston, N. Zotov, and M. Elaasar, "A BalloonBorne Coded Aperture Telescope for Arc-Minute Angular Resolution at Hard X-Ray Energies," Proc. SPIE 2806, pp. 349-360, 1996.

7. M. L. Cherry, P. P. Altice, D. L. Bnad, J. H. Buckley, G. T. Guzik, P. L. Hink, S. C. Kappadth, J. R. Macri, J. L. Matteson, M. L. McConnell, T. J. O’Neill, J. M. Ryan, K. R. Slavis, J. G. Stacy, and A. D. Zych, "Coded-aperture Xray/gamma-ray telescope for arc-minure localizations of gamma-ray bursts, “ Proc. SPIE 3765, pp. 539-550, 1999.

8. D. Bhattacharya, D.D. Dixon, V. Kong, T.J. O'Neill, R.S. White, A.D. Zych, J.Ryan, M. McConnell, J. Macri, J. Samimi, A. Akyuz, W.A. Mahoney, L.S. Varnell, "A Tracking and Imaging Gamma-Ray Telescope (TIGRE) for Energies of 0.3 to $100 \mathrm{MeV}$," Proc. 26th Internat. Cosmic Ray Conf., Salt Lake City, 5, pp. 72-75, 1999,.

9. J. M. Ryan, J. R. Macri, M. L. McConnell, B. K. Dann, M. L. Cherry, T. G. Guzik, F. P. Doty, B. A. Apotovsky, J. F. Butler, "Large Area Sub-millimeter Resolution CdZnTe Strip Detector for Astronomy," Proc. SPIE 2518, pp. 292-301, 1995.

10. J. R. Macri, D. V. Boykin, K. Larson, M. Mayer, M. L. McConnell, J. M. Ryan, M. L. Cherry, T. G. Guzik, B. A. Apotovsky, J. F. Butler, F. P. Doty, and C. L. Lingren, "Progress in the Development of Large Area Sub-millimeter Resolution CdZnTe Strip Detectors," Proc. SPIE 2859, pp. 29-36, 1996.

11. J. R. Macri, B. A. Apotovsky, J. F. Butler, M. L. Cherry, B. K. Dann, A. Drake, F. P. Doty, T. G. Guzik, K. Larson, M. Mayer, M. L. McConnell, and J. M. Ryan, "Development of an Orthogonal-Stripe CdZnTe Gamma Radiation Imaging Spectrometer," IEEE Trans. Nucl. Sci. 43, 1458, 1996. 
12. M. Mayer, D. V. Boykin, M. L. Cherry, J. F. Courville, F. P. Doty, A. Drake, T. G. Guzik, L. A. Hamel, K. Larson, J. R. Macri, M. L. McConnell, ,J. M. Ryan, O. Tousignant, "Performance and Simulation of CdZnTe Strip Detectors as Suibmillimeter Resolution Imaging Gamma Radiation Spectrometers," IEEE Trans. Nucl. Sci. 44, 922, 1997.

13. O. Tousignant, L.-A. Hamel, J.-F. Courville, P. Paki, J. R. Macri, K. Larson, M. Mayer, M. L. McConnell, and J. M. Ryan, "Progress in the study of CdZnTe strip detectors," Proc. SPIE 3115, pp. 214-224, 1997.

14. L. A. Hamel, O. Tousignant, J. F. Courville, J. R. Macri, K. Larson, M. Mayer, M. L. McConnell, and J. M. Ryan, "An imaging CdZnTe detector with orthogonal anodes", Proceedings of the 1997 Fall Meeting of the Material Research Society, Boston, December (1997).

15. V. T. Jordanov, J. R. Macri, and M. L. Mayer, "Biasing Scheme for AC Coupled Strip Detectors", IEEE Trans. Nucl. Sci. 45(3), pp. 787-791, 1998.

16. M. Mayer, L. A. Hamel, O. Tousignant, J. R. Macri, J. M. Ryan, M. L. McConnell, V. T. Jordanov, J.F. Butler, and C.L. Lingren, "Signal Formation in a CdZnTe Imaging Detector with Coplanar Pixel and Control Electrodes," NIM A422, pp 190-194, 1999.

17. O. Tousignant, L. A. Hamel, P. Dufor, F. Joly, J. R. Macri, M. L. McConnell, J. M. Ryan, and V. T. Jordanov, "Energy and position resolution of a CdZnTe gamma-ray detector with orthogonal coplanar anodes." Proc. SPIE 3768, pp. 38-48, 1999.

18. K.A. Larson, L. Hamel, V. Jordanov, J.R. Macri, M.L. McConnell, J.M. Ryan, O. Tousignant, and A. Vincent, "The Development of a Position-Sensitive CZT Detector wth Orthogonal Co-Planar Anode Strips", Fifth Compton Symposium, eds. M.L. McConnell \& J.M. Ryan, AIP Conf. Proc. 510, pp. 774-778, AIP, New York, 2000.

19. Z. He, W. Li, G. F. Knoll, D. K. Wehe, J. Berry, and C. M. Stahle, "3-D position sensitive CdZnTe gamma-ray spectrometers," NIM A422, pp. 173-178, 1999.

20. J. L. Matteson, F. Duttweiler, G. L. Huszar, P. C. Leblanc, R. E. Skelton, E. A. Stephan, P. L. Hink, P. F. Dowkontt, K. R. Slavis, T. O. Tumer, and S. D. Kavis, "Position-sensitive CZT detector module," Proc. SPIE 3446, pp 192-201, 1998.

21. V. T. Jordanov, J. R. Macri, J. E. Clayton, and K. A. Larson, "Multi-Electrode CZT Detector Packaging Using Polymer Flip Chip Bonding", 11th International Workshop on Room Temperature Semiconductor X- and Gamma-Ray Detectors and Associated Electronics, Vienna, Austria, October (1999), to be published in NIM A.

22. F. W. Kulesza, and R. H. Estes, Advanced Packaging, Nov (1997) 26.

23. LeClair, T., et al., Proc.Int. Symp. on Microelectronics (IMAPS), (1998) 758.

24. K. Larson, V. Jordanov, M. L. McConnell, J. R. Macri, J. M. Ryan, L.-A., Hamel, and O. Tousignant, "Analog Processing of Signals from a CZT Strip Detector with Orthogonal Coplanar Anodes," to be published in Proc. SPIE 4141, 2000. 